\title{
"Thermal-Micromechanics" coupling approach to model the corrosion and crack of concrete anchor
}

\author{
Zhixiong Chen ${ }^{1,2^{*}}$, Xiaodong Pan ${ }^{1,2}$, Chengqian Lin ${ }^{1,2}$, Hao Huang ${ }^{1,2}$, Liang \\ $\mathrm{Lu}^{1,2}$
}

\begin{abstract}
${ }^{1}$ Key Laboratory of New Technology for Construction of Cities in Mountain Area (Chongqing University), Ministry of Education, Chongqing 400045, China

${ }^{2}$ School of Civil Engineering, Chongqing University, Chongqing 400045, China

Correspondence should be addressed to Zhixiong Chen; chenzhixiong@cqu.edu.cn
\end{abstract}

Keywords: "Thermal-Micromechanics" coupling approach; Discrete element method; Stress corrosion; Diffusion of the corrosive media; Anchor

\begin{abstract}
A "Thermal-Micromechanics" coupling approach for corrosion and crack of anchor in the corrosive environment is researched in this study. The thermal module of software PFC2D is applied to simulate diffusion of corrosive media in the concrete while the mechanics module of PFC2D is applied to model stress corrosion and crack resulting from long term tension stress. Information related to the concentration of corrosive media is transferred into the mechanics module to modify the initial crack stress. Bond-fracture information is then read by the thermal module to change the concentration in the crack position. Comparison with experimental data indicates this coupling approach may model diffusion of the corrosive media and the crack satisfactorily.
\end{abstract}

\section{Introduction}

Anchors were widely applied as permanent support in geotechnical reinforcement engineering projects requiring critical reliability including reinforcing hydraulic dams, supporting slopes, preventing landslides, large underground caverns and deep foundation pits [1].

A corrosive medium often exists in the working environment of these structures that are often characterized by complex details, exceptional working stress, quality control issues, and hidden operation in construction and maintenance, thus rendering corrosion as a key problem. Thirty five cases of anchor cable or bolt fracture resulting from corrosion have been collected since 1986 by the anchor working group Fédération Internationale de la Précontrainte (FIP) [2] along with several individual failure cases also reported according to other sources.

\section{The diffusion of the corrosive media in the concrete}

Diffusion is the main corrosive media transmission mode inside concrete and extensive research has been conducted related to it. Numerous experiments demonstrate the diffusion process of common corrosive media in concrete is a linear one-dimensional diffusion process and may be described utilizing Fick's second law, as follows:

$$
\left\{\begin{array}{l}
\partial C / \partial t=D \partial^{2} C / \partial x^{2} \\
C(0, t)=C_{s} \\
C(x, 0)=C_{0}
\end{array}\right.
$$


Where $\mathrm{C}_{0}$ is the initial concentration of corrosive media inside the concrete; $\mathrm{C}_{\mathrm{s}}$ is the initial concentration of corrosive media on the concrete surface; $\mathrm{D}$ is the diffusion coefficient; $\mathrm{x}$ is the distance to the concrete surface, and $t$ is the immersion time.

The Particle Flow Code, as the simplified discrete element method, was first proposed by Cundall[3] and was widely utilized for analysis of rock mechanics. The program employs the discrete element method to simulate movement and interaction of circular particulate media. Translational and rotational displacement may also be modeled, allowing separation of the existing contact and formation of the new primary contact between particles, thus inherent advantages exist for simulated crack development. Thermal diffusivity of material is primarily defined by the three parameters of density, thermal conductivity and specific heat as:

$$
a=\frac{\lambda}{c \rho}
$$

To match computing of the diffusion process with computing of the temperature transformation, the diffusion coefficient is replaced by thermal conductivity while material density and specific heat were assigned 1 in the $\mathrm{PFC}^{2 \mathrm{D}}$ analysis process.

\section{Stress corrosion of the concrete anchor}

$\mathrm{PFC}^{2 \mathrm{D}}$ typically utilizes the parallel bonded mode (BMP) to model concrete and rock. The PSC model assumes that when stress exceeds threshold value for crack development $\left(\sigma_{a}\right)$ and is less than the bond strength, radius of the parallel bond will be decreased with time according to an exponential role[4]. When the stress exceeds strength, rupture will occur in the bond, and the visible crack will appear. Decreasing of the radius utilizes the following rule:

$$
V=\left\{\begin{array}{lc}
0, & \bar{\sigma}<\bar{\sigma}_{a} \\
\beta_{1} e^{\beta_{2}\left(\bar{\sigma} / \bar{\sigma}_{c}\right)}, & \bar{\sigma}_{a} \leq \bar{\sigma}<\bar{\sigma}_{c} \\
\infty, & \bar{\sigma} \geq \bar{\sigma}_{c}
\end{array}\right.
$$

Addition of only three parameters is required by the PSC model (Eq. 4) to describe the stress corrosion process: $\beta_{1}, \beta_{2}$ and $\sigma \mathrm{c} . \beta_{1}$ and $\beta_{2}$ are parameters related to temperature and concentration.

Groups of the cement mortar anchor were immersed in chlorine ion solution with different concentration $(5 \%, 8 \%, 12 \%, 15 \%)$ to study the diffusion process of corrosive media. A PFC ${ }^{2 \mathrm{D}}$ model was established to study the influence of stress corrosion on concrete anchors under long-term stress[5] (Fig. 1). A tension force was applied to the steel anchor bar through a pre-compressed spring. Impact of tension stress magnitude was studied with different tension forces applied as $0.6 \mathrm{Pu}$ and $0.3 \mathrm{Pu}$, where $\mathrm{Pu}$ was calculated utilizing tension strength of the concrete and $\mathrm{Pu}=791 \mathrm{~N}$. The $\mathrm{PFC}^{2 \mathrm{D}}$ model featured a uniform particle diameter of $0.5 \mathrm{~mm}$ and 12732 balls in total.

Tab. 1 The parameters using in the $\mathrm{PFC}^{2 \mathrm{D}}$ model

\begin{tabular}{cccccc}
\hline & $\begin{array}{c}\text { Size } \\
(\mathrm{mm})\end{array}$ & $\begin{array}{c}\text { Shear stiffness } \\
\mathrm{Pa}\end{array}$ & $\begin{array}{c}\text { Normal stiffness } \\
\mathrm{Pa}\end{array}$ & $\begin{array}{c}\text { Frictional } \\
\text { Coefficient }\end{array}$ & Damping ratio \\
\hline Ball & 0.5 & $1 \mathrm{e} 9$ & $1 \mathrm{e} 9$ & 1.0 & \\
Anchor Bolt & 5 & $1 \mathrm{e} 9$ & $1 \mathrm{e} 9$ & 1.0 & 0.3 \\
Wall & - & & $1 \mathrm{e} 11$ & 1.0 & \\
\hline
\end{tabular}

The parameters used for the stress corrosion are listed below and in Tab. 1:

Tensile strength in the parallel bond $\mathrm{pb}$ _ten $=3.0 \mathrm{e} 6 \mathrm{~Pa}$

Shear strength in the parallel bond $\mathrm{pb} \_$ten $=3.0 \mathrm{e} 6 \mathrm{~Pa}$ 
Threshold of stress for crack to develop: $\sigma_{0}=100 \mathrm{~Pa}$

Parameters for stress corrosion: $\beta_{1}=2.5, \beta_{2}=30$ ( David O. Potyondsy [4])

\section{"Thermal-Micromechanics" coupling approach to model the corrosion and crack of anchor}

The diffusion process of the corrosive media inside the concrete anchor may be studied utilizing the thermodynamics framework and the micro-cracks from long-term stress may be analyzed by the fracture mechanics theory as mentioned before. Microscopic cracks that originated from long-term stress accelerate invasion of corrosive media and the invasion of corrosive media then acts to deteriorate the concrete through a mutual coupling process that includes decreasing the cracks opening strength threshold and further accelerating crack occurrence. $\mathrm{PFC}^{2 \mathrm{D}}$ thermal module outputs the distribution of chloride ions concentration in the model at the end of each sub-step. The mechanical module reads this information and revises the crack opening strength to then produce a fractures layout in the model.

A strength reduction factor is introduced in this study to consider the influence of corrosive medium on the deterioration effect of concrete and to account for the concentration of chloride ions on the strength threshold of crack opening as:

$$
\mathrm{R}_{c l}=1-\left(\mathrm{t}-\mathrm{t}_{0}\right) \cdot \mathrm{A}
$$

Where $\mathrm{t}$ is the ion concentration in the specific location; $\mathrm{A}$ and $\mathrm{t}_{0}$ are coefficients. By fitting the experimental data, these coefficients are $A=0.9, t_{0}=0.1 \%$. If $\mathrm{R}_{\mathrm{cl}}<0.1$ then, $\mathrm{R}_{\mathrm{cl}}=0.1$.
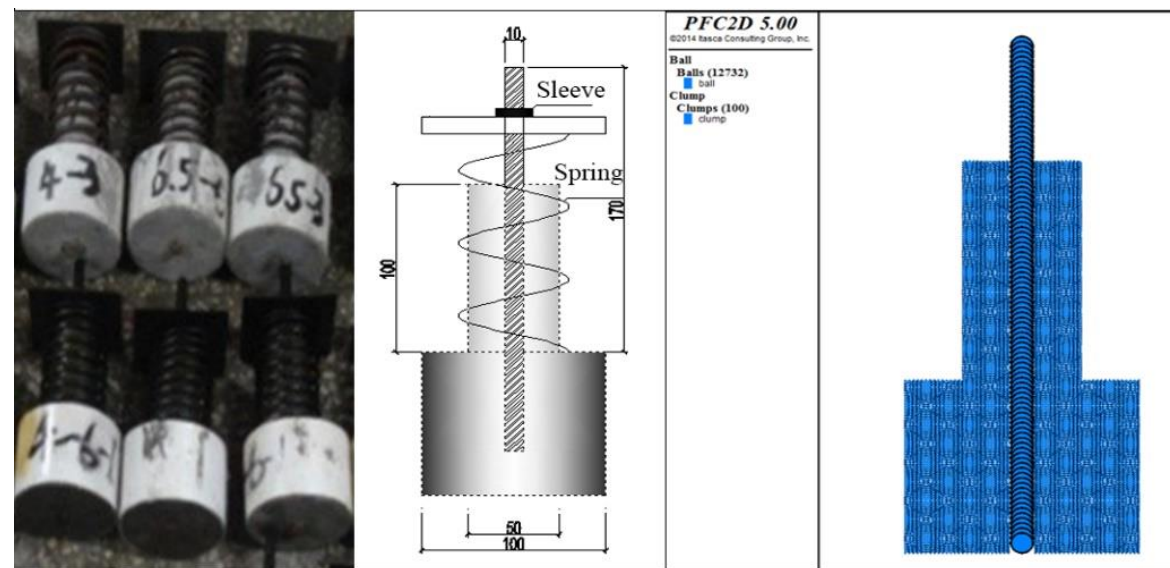

Fig. 1 The specimen with long-term tension (left) and its size (middle) and the $\mathrm{PFC}^{2 \mathrm{D}}$ model (right).

The number of fractures increase to 80 from 14 when only stress corrosion is considered and may be explained as, with the increase of immersion time, the chloride ion concentration near the surface of the sample increases, reducing the cracking strength threshold of concrete. Chloride ions concentration in the crack zone is also observed to increase, consistent with the observation that when the specimen cracks, the corrosive solution will follow the crack into the interior of the specimen, leading to further concentration increases in that region.(Fig. 2)

Fig. 3 displays simulation results of normal stress in the parallel bond for stress corrosion $\mathrm{t}=12$ month. Depth of cracks formed by parallel bond breakage is nearly twice that of when only stress corrosion is considered. Maximum tension stresses are still at the crack tip zone, with maximum value of $6474 \mathrm{~Pa}$, approximately one-third smaller than $9721 \mathrm{~Pa}$ when only stress corrosion is considered.

Fig. 4 displays the distribution of Chloride ions concentration of simulation and experimental results for $0.6 \mathrm{Pu}+15 \% \mathrm{NaCl}$ and $\mathrm{t}=12$ month. Chloride ion concentration at $1.5 \mathrm{~mm}$ distance to the surface is $0.87 \%$ for the simulation and $0.8 \%$ for the experiment. If stress corrosion only is considered, 
corresponding results are then $0.73 \%$ for simulation and $0.65 \%$ for experiment. Increase in concentration after considering the coupling effect is a result of long-term tension stress applied to the sample causing the tension crack and intrusion of corrosive media, thus concentration increases for the same location.

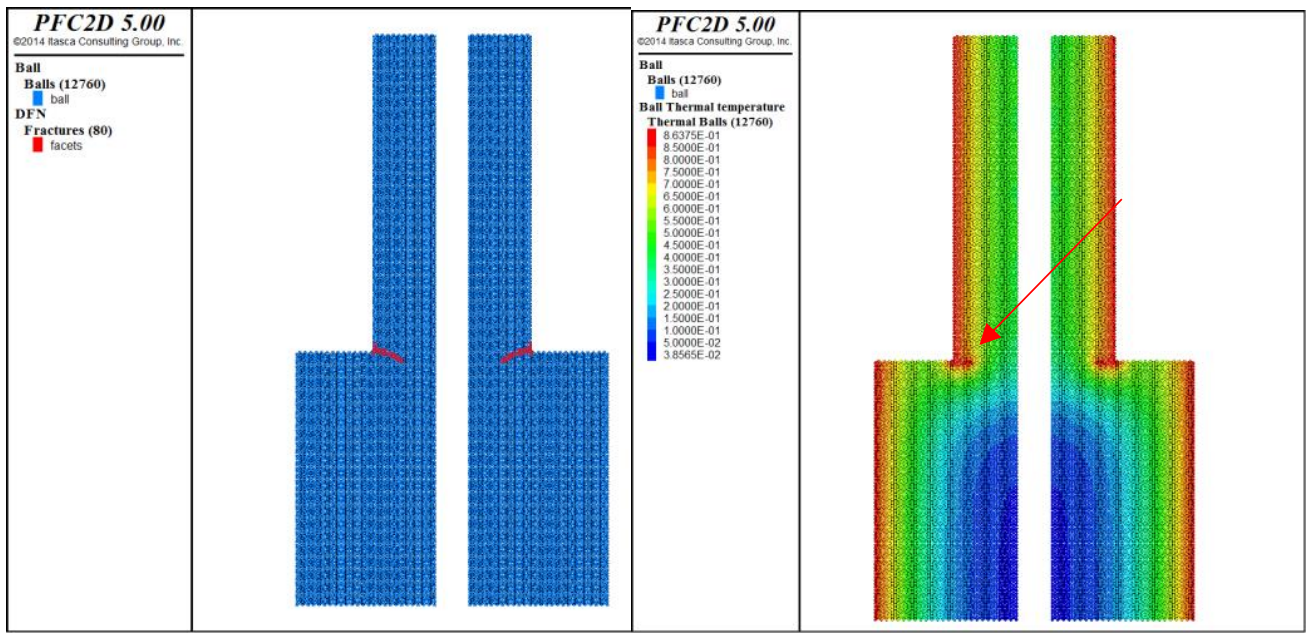

Fig. 2 The cracks of stress corrosion (left) for $\mathrm{t}=12$ month and the distribution of chloride ions concentration (right)
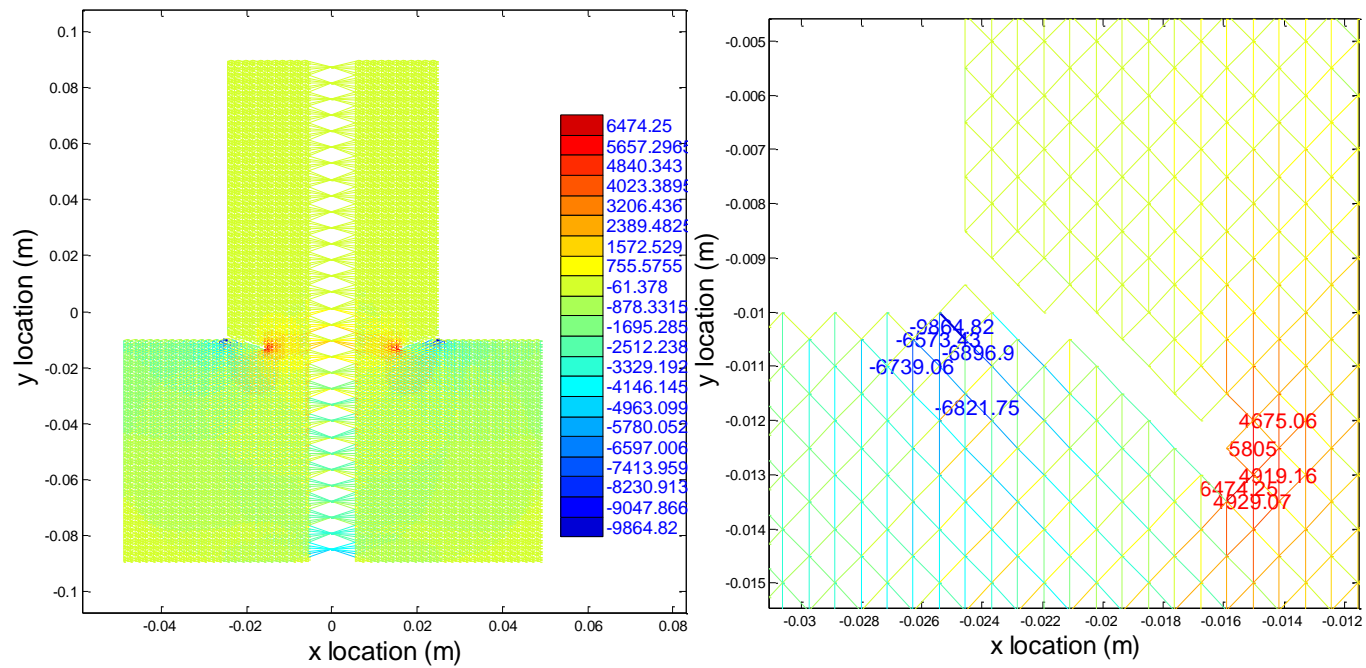

Fig. 3 The normal stress in the parallel bond for stress corrosion $t=12$ month

As shown in Fig. 5, Maximum normal stress in X direction increases to $2000 \mathrm{~Pa}$ after the coupling calculation, with the value at $1000 \mathrm{~Pa}$ when only stress corrosion is considered. The maximum normal stress in $\mathrm{Y}$ direction increases to 4000 from $3100 \mathrm{~Pa}$ for both the coupling calculation and the stress corrosion only calculation. Location of the maximum normal stress in $\mathrm{Y}$ direction changes from $17.5 \mathrm{~mm}$ distance to the bolt surface to $7.5 \mathrm{~mm}$ after the coupling calculation indicating that stress redistribution occurs with the crack opening process. 

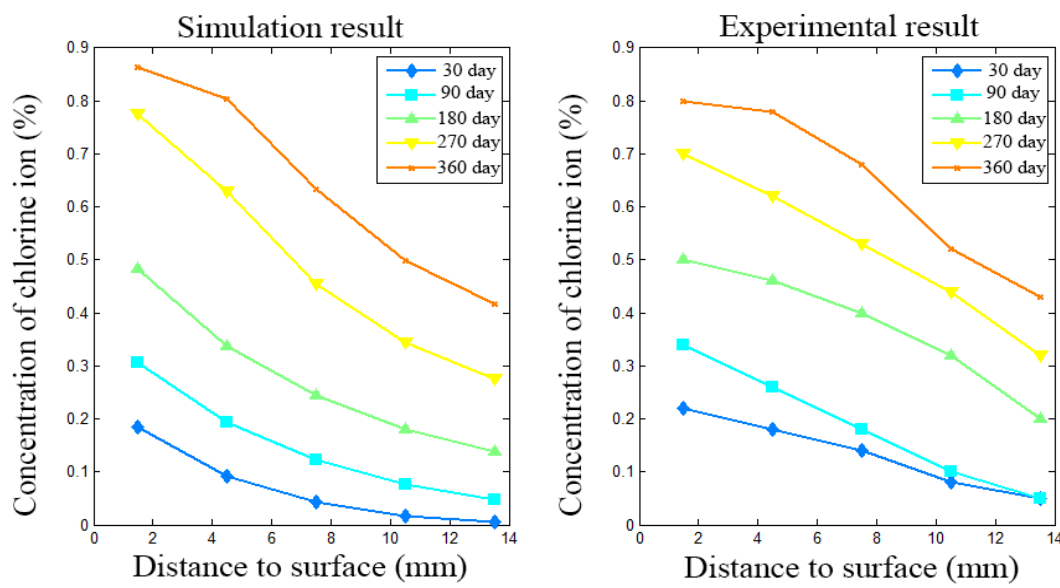

Fig. 4 Distribution of Chloride ions concentration for $0.6 \mathrm{Pu}+15 \% \mathrm{Nacl}$
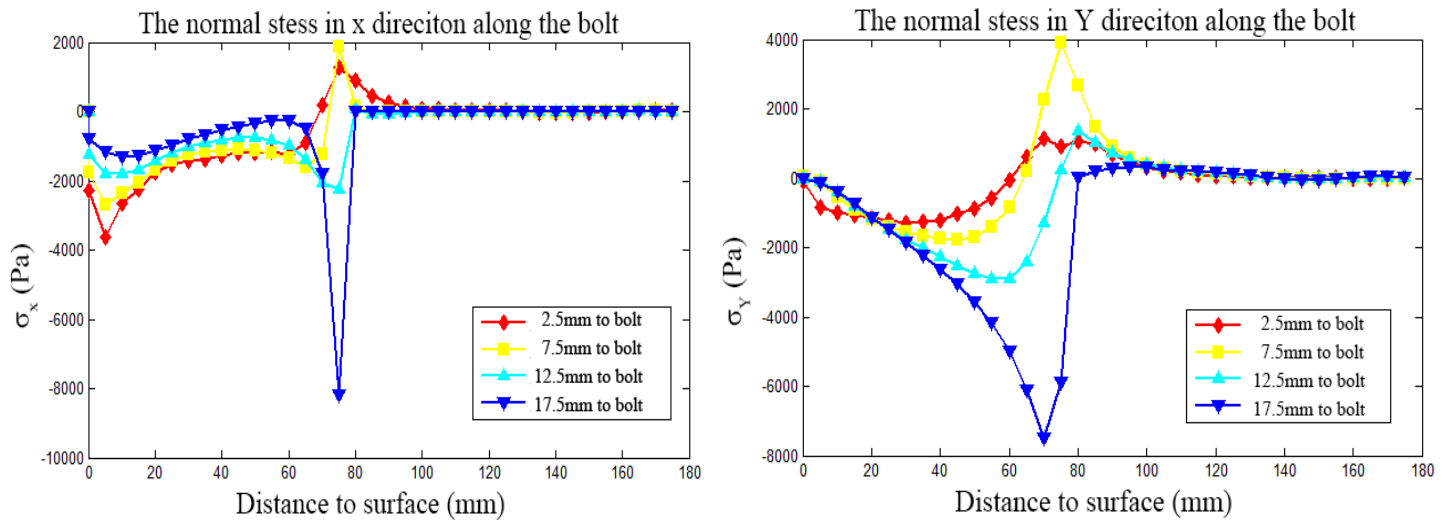

Fig. 5 Normal stress in the $\mathrm{X}$ and $\mathrm{Y}$ direction for $\mathrm{t}=12$ month of coupling corrosion

\section{Conclusions}

The stress corrosion theory may be utilized to study time-dependent weakening processes that occur in concrete anchor PSC mode is applied in this study to simulate the cracking opening process and reveals that fractures occur in the high tension stress zone and, following crack occurrence, stress inside the anchor will re-distribute. A coupling model is developed in the study to analyze the complex process of the concrete anchor deterioration. The PFC2D thermal modules and mechanical modules are applied respectively to simulate the chemical corrosion and stress corrosion process. Results reveal the number and the depth of fractures increase and the maximum stress decreases, similar to the experimental data and also credible.

\section{Acknowledgements}

This work was financially supported by the International Cooperation and Exchanges of NSFC, China (Grant No. 5141001028), National Natural Science Foundation of China (Grant No. 51308566, 51778092, 51408081) and the Fundamental Research Funds for the Central Universities of China (Grant No. 106112016CDJXZ208808).

\section{References}

[1] John G, Littlejohn S. Durability of buried and encased ground anchorages and structural components for corrosivity assessment and protection to ensure service life. In: International conference on ground anchorages and anchored structures in service 2007; London, UK.

[2] FIP State-of-Art Report. Corrosion and corrosion protection of prestressed ground anchorages. 
Fédération Internationale de la Précontrainte 1986, London.

[3] CUNDALL P A, STRACK O D L. A discrete numerical model for granular assemblies[J]. Geotechnique, 1979, 29(1): 47-65.

[4] David O. Potyondy. Simulating stress corrosion with a bonded-particle model for rock. International Journal of Rock Mechanics \& Mining Sciences. 2007, 44: 677-691.

[5] Jian Liang. Research on durability of bolt anchor structure based on multiple coupling factors[D]. ChongQing, China. ChongQing University, 2016. 